\title{
VLT spectrosocpy of RR Lyrae stars in the Sagittarius northern tidal stream
}

\author{
S. Duffau ${ }^{1}$, M. T. Ruiz ${ }^{1}$, R. Zinn ${ }^{2}$ and A. K. Vivas ${ }^{3}$ \\ ${ }^{1}$ Departamento de Astronimia, Universidad de Chile \\ Casilla 36-D, Santiago, Chile \\ email: sduffau@das.uchile.cl, mtruiz@das.uchile.cl \\ ${ }^{2}$ Department of Astronomy, Yale University, \\ P.O. Box 208101,New Haven, CT-06520-8101, USA \\ email: zinn@astro.yale.edu \\ ${ }^{3}$ Centro de investigaciones de Astronomia, Apartado Postal 264, Merida 501-A, Venezuela, \\ email: akvivas@cida.ve
}

\begin{abstract}
We present VLT spectroscopy of 13 RR Lyrae variables from the QUEST survey that lie in the leading arm of the tidal stream from Sagittarius dSph galaxy. We observed these stars to measure their radial velocities and metal abundances and to complete a sample of stars from the overdensity detected by QUEST in this area of the sky. The full sample contains 42 stars, 29 observed with VLT and 13 observed with GEMINI, we report here the results from the VLT observations so far. We conclude that the new stars and stars reported before by Vivas, Zinn and Gallart (2005) are consistent with belonging to Sgr. We discuss the importance of the final third of the sample, the GEMINI targets, which will provide important constrains on models of the Sgr streams that assume different dark matter halo shapes.
\end{abstract}

Keywords. Galaxy: formation, Galaxy: halo.

\section{Introduction}

A detailed study of the Sgr. tidal tails may help us understand Galaxy formation in many ways. Sgr is the prototypical case of the process of tidal disruption and galaxy merger, providing insight on a process that might be responsible for the formation of the luminous halos of galaxies like our own. The stellar populations along the tails might have evolved under the presence of the strong tidal forces, this can be revealed by comparing different stellar populations at different location on the streams. Finally the streams have been proposed as possibly powerful tools to study the shape of the Milky Ways potential, which at large galactocentric distances (like the ones involved in this study), may be dominated by the shape of the dark matter halo (DM). Theoretical models of Sgr tidal disruption under assumptions of different DM halo shapes differ on small scales. The precisions that are necessary to distinguish between these small differences observationally are achieved with RR Lyrae stars because they are excellent standard candles providing their location in space very precisely, much more than with other tracers. The ultimate goal of this research is to test models of the streams that assume different shapes for the gravitational potential of the MW, using both the high positional accuracy of RR Lyrae stars and their radial velocities. For the moment we have studied two thirds of the intended sample using VLT. GEMINI data on the last third will follow.

\subsection{Metal Abundances and Radial Velocities}

Metal abundances were determined using a modified version of the $\Delta S$ method developed by Layden. Stars in our sample concentrate at metal abundances between -2.0 and -1.5 


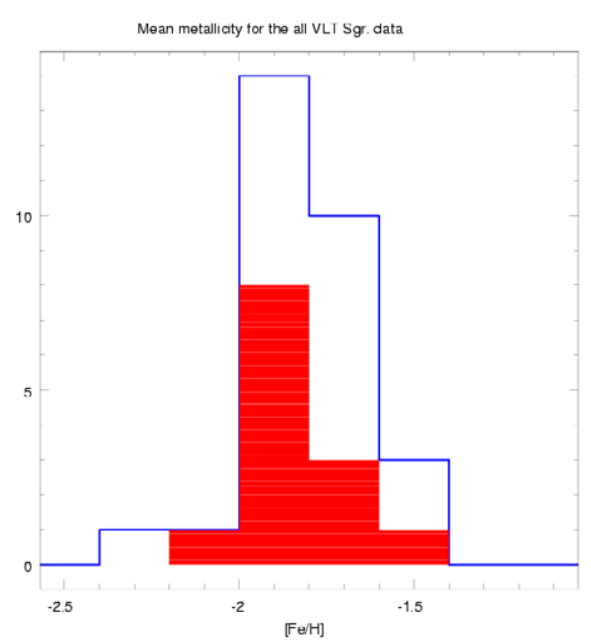

Figure 1. Metal Abundances compared, see text.

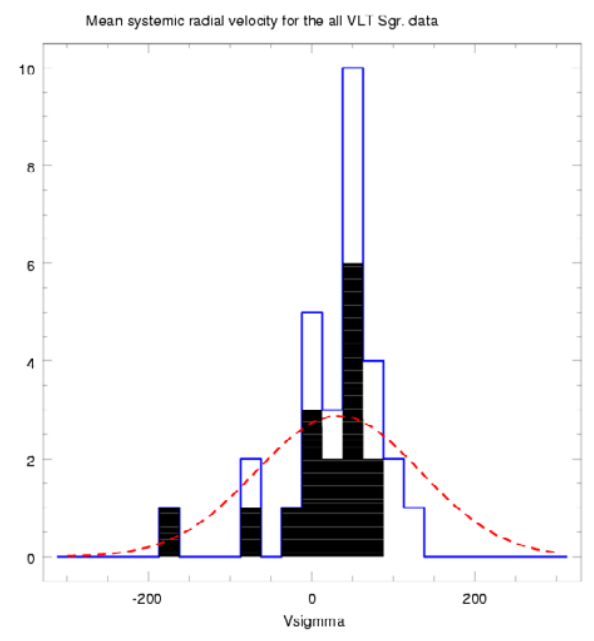

Figure 2. Velocities compared, see text.

in the Zinn \& West Globular Cluster metallicity scale. The mean value of the metalicity of the stars in the main peak is now -1.8 , compared to the previous result of -1.76 . You can see the contribution of our work to the sample analized up until now displayed on the histogram of figure 1, red is only data from this work. The blue profile is the sum of the two contributions. After adding our data, the velocity histogram becomes figure 2 . in blue you see the profile of the histogram for all 29 stars observed with VLT. In solid black you see the original histogram from Vivass work. The new mean value for the stars in the main peak is $44 \mathrm{~km} / \mathrm{s}$, compared with $33 \mathrm{~km} / \mathrm{s}$ for the Vivas sample only. The dashed red line represents the smooth gaussian distribution of 29 random halo stars in the same area of the sky. As this is an overdense region, significantly fewer RR Lyrae stars were expected to lie in this region.

\subsection{Conclusions}

We conclude that the new measurements are consistent with the previous ones. When combined with them, more robust results are obtained for the mean velocity, the velocity dispersion, and the metallicity distribution of the RR Lyrae variables in the northern stream of Sgr. We await the results from the last third of the total sample of targets to complete the study and define the extent in space and the mean values of radial velocity and metal abundance and their dispersions properly.

\section{Acknowledgements}

We would like to acknowledge support from the following sources: ALMA-Sochias fellowship, NSF AST-05-07364, IAU grant, FONDAP, MECESUP and Fundacin Andes. We want to thank R. A. Mendez who was PI of the latest VLT proposal.

\section{Reference}

Vivas, A. K., Zinn, R. \& Gallart, C. 2005, Astrophysical Journal 129, 189 